\title{
The NRF2-PGC-1 $\beta$ pathway activates kynurenine aminotransferase 4 via attenuation of an E3 ubiquitin ligase, synoviolin, in a cecal ligation/perforation-induced septic mouse model
}

\author{
YUSUKE ISHIDA $^{1 *}$, HIDETOSHI FUJITA $^{2,3^{*}}$, SATOKO ARATANI $^{2,3}$, MIYUKI CHIJIIWA $^{1}$, \\ NOBORU TANIGUCHI ${ }^{4}$, MAHO YOKOTA ${ }^{2}$, YUKIHIKO OGIHARA ${ }^{1}$, NAOMI UOSHIMA $^{1}$, \\ FUMIAKI NAGASHIMA $^{1}$, HIROYUKI UCHINO ${ }^{1}$ and TOSHIHIRO NAKAJIMA ${ }^{2,3,5-10}$
}

${ }^{1}$ Department of Anesthesiology, Tokyo Medical University Hospital, Tokyo 160-0023; ${ }^{2}$ Institute of Medical Science, ${ }^{3}$ Department of Future Medical Science, Tokyo Medical University, Tokyo 160-8402; ${ }^{4}$ Department of Medicine of Sensory and Motor Organs, Division of Orthopedic Surgery, Faculty of Medicine, University of Miyazaki, Kiyotake, Miyazaki 889-1692; ${ }^{5}$ Integrated Gene Editing Section (iGES); ${ }^{6}$ Medical Research Center; ${ }^{7}$ Industry-University Cooperation

(BioMimetics Sympathies Inc.), Tokyo Medical University Hospital, Tokyo 160-0023; ${ }^{8}$ Institute of Medical Science, St. Marianna University School of Medicine, Kawasaki, Kanagawa 216-8511; ${ }^{9}$ Department of Biomedical Engineering, Osaka Institute of Technology, Osaka 535-8585; ${ }^{10}$ Bayside Misato Medical Center, Kochi 781-0112, Japan

Received June 15, 2017; Accepted March 15, 2018

DOI: $10.3892 / \mathrm{mmr} .2018 .9175$

\begin{abstract}
Sepsis-associated encephalopathy (SAE) is a systemic inflammatory response syndrome of which the precise associated mechanisms remain unclear. Synoviolin (Syvn1) is an E3 ubiquitin ligase involved in conditions associated with chronic inflammation, including rheumatoid arthritis, obesity, fibrosis and liver cirrhosis. However, the role of Syvn1 in acute inflammation is not clear. The aim of the present study was to investigate the role of Syvn1 in a septic mouse model induced by cecal ligation/perforation (CLP). Metabolome analysis revealed that kynurenine (KYN), a key factor for the development of neuroinflammation, was increased in CLP-induced septic mice. Notably, KYN was not detected in CLP-induced
\end{abstract}

Correspondence to: Professor Toshihiro Nakajima, Institute of Medical Science, Tokyo Medical University, 6-1-1 Shinjuku, Shinjuku, Tokyo 160-8402, Japan

E-mail: marlin@tokyo-med.ac.jp

${ }^{*}$ Contributed equally

Abbreviations: Syvn1, synoviolin; SAE, sepsis-associated encephalopathy; CLP, cecal ligation/perforation; KYN, kynurenine; KYNA, kynurenic acid; KATs, kynurenine aminotransferases; PGC-1 $\beta$, Peroxisome proliferator-activated receptor coactivator $1 \beta$; QA, quinolinic acid; 3-HAA, 3-hydroxykynurenine; SPF, specific pathogen-free

Key words: synoviolin, NRF2, PGC-1 1 , kynurenine, septic mouse model, kynurenine aminotransferase 4, E3 ligase septic Syvn1-deficient mice. KYN is converted to kynurenic acid (KYNA) by kynurenine aminotransferases (KATs), which has a neuroprotective effect. The expression of KAT4 was significantly increased in Syvn1-deficient mice compared to that in wild-type mice. Promoter analysis demonstrated that Syvn1 knockdown induced the KAT4 promoter activity, as assessed by luciferase reporter activity, whereas Syvn1 overexpression repressed this activity in a dose-dependent manner. Furthermore, the KAT4 promoter was significantly activated by the transcriptional factors, NF-E2-related factor 2 and peroxisome proliferator-activated receptor coactivator $1 \beta$, which are targets of Syvn1-induced degradation. In conclusion, the results of the current study demonstrates that the repression of Syvn1 expression induces the conversion of neurotoxic KYN to neuroprotective KYNA in a CLP-induced mouse model of sepsis, and that Syvn1 is a potential novel target for the treatment of SAE.

\section{Introduction}

Sepsis is a form of systemic inflammation caused by an infection, and multiple organ failure is known to occur when this condition intensifies. Clinical studies demonstrated that the central nervous system might be one of the first organs affected by sepsis. Sepsis-associated encephalopathy (SAE), characterized by diffuse cerebral dysfunction, is secondary to sepsis and is related to increased morbidity and mortality $(1,2)$. Clinical symptoms of SAE are delirium, fluctuating changes in mental status, lack of attention, and disorganized thinking. Encephalopathy of variable severity occurs in $9-71 \%$ of septic patients, and those with central nervous disorders have the worst prognosis in terms of cognitive and motor function. 
In addition, it has been reported that the mortality rate of septic patients with SAE is approximately twice that of septic patients without SAE; moreover, the mortality rate increases to $63 \%$ when patients with SAE present with a Glasgow Coma Scale (GCS) value of 3-8 (1,2). Although several mechanisms including inflammation or the disturbance of neurotransmission disturbance, have been proposed, the precise mechanisms responsible for sepsis-induced cognitive impairment have not been fully elucidated.

The kynurenine (KYN) pathway is well known to be a major mechanism of tryptophan catabolism, and it is activated during neuroinflammation during several neurodegenerative diseases $(3,4)$ such as SAE $(5)$. Tryptophan is converted to $\mathrm{KYN}$, and $\mathrm{KYN}$ is converted into three intermediates, quinolinic acid (QA), 3-hydroxykynurenine (3-HAA), and kynurenic acid (KYNA) via two different pathways (Fig. 1). 3-HAA and QA are neurotoxic, whereas KYNA is neuroprotective. The relative balance between the two branches of this pathway might play an important role in the development of neuroinflammation. The conversion to KYNA is catalyzed by kynurenine aminotransferases (KATs), which have been detected in the brain and peripheral tissues such as the skeletal muscle $(6,7)$. Recent studies showed that the peroxisome proliferator-activated receptor (PPAR)-PPAR coactivator-1 (PGC-1) pathway induces skeletal muscle KAT expression during exercise $(7,8)$. The analysis of PGC-1 $\alpha 1$ skeletal muscle-specific transgenic mice showed that increased expression of skeletal muscle KATs induced KYN metabolism. Synthesis of KYNA was enhanced and the accumulation of KYN was reduced, thereby protecting against stress-induced depression (7).

Among several hundreds of E3 ubiquitin ligases, synoviolin (Syvn1), identified from the cDNA of rheumatoid synovial cells, is the only known regulator of PGC-1 $\beta$ ubiquitination (9). We recently demonstrated that Syvn1 interacts with PGC-1 $\beta$ and induces its degradation (9). Global elimination of Syvnl in post-neonatal mice is associated with weight loss and reduced white adipose tissue through enhanced energy expenditure, which is mediated by the function of PGC-1 $\beta$ (9). PGC-1 $\beta$ and PGC-1 $\alpha$ share extensive sequence identity to each other $(10,11)$, and the primary structure of PGC-1 $\beta$ has several unique features of primary structure such as LXXLL in its middle portion and the absence of a proline-rich region at the C-terminus. Knockout studies have also suggested functional differences between PGC- $1 \alpha$ and PGC-1 $\beta$, such as lethality (12). We previously demonstrated that Syvn1 is involved in the development of rheumatoid arthritis, fibrosis, limb girdle muscular dystrophy, and liver cirrhosis (13-17). We also described another unique function of Syvn1; specifically, Syvn1 entraps and degrades tumor suppressor p53 and NRF2 (17,18). Nrf2 is a transcriptional factor that activates antioxidant and cytoprotective genes that share a common a cis-acting enhancer sequence, termed the antioxidant response element (ARE), under conditions of oxidative stress. Several studies indicate that Nrf2 is neuroprotective against neurotoxicity (19-21). These results suggest that loss of Syvn1 expression might have neuroprotective effects.

In the present study, we therefore investigated whether Syvn1-deficient mice were resistant to cecal ligation/perforation (CLP) treatment, which is a mouse model of sepsis, and revealed that Syvn1 ablation mediates the activation of tryptophan metabolism via KAT4 gene expression.

\section{Materials and methods}

Mice. All procedures involving animals were performed in accordance with institutional and national guidelines for animal experimentation, and were approved by the Institutional Animal Care and Use Committee of Tokyo Medical University (\#S-24021). Mice were kept in specific pathogen free (SPF) under standard conditions $\left(20-26^{\circ} \mathrm{C}\right.$ temperature; $40-65 \%$ humidity) with a 12-h light/12-h dark cycle. F-1 Foods (5.1\% fat, $21.3 \%$ protein) were purchased from Funabashi farm (Chiba, Japan). All mice used in the study were of the C57BL/6J background. Heterozygous Syvn1 mice and tamoxifen (Tam)-inducible Syvnl knockout mice were previously described $(9,13)$.

Sepsis model. Male C57/BL6 mice at 7-9 weeks of age were anesthetized with $4-5 \%$ sevoflurane and a middle abdominal incision was made along the ventral surface of the abdomen to expose the cecum. Before perforation, feces were gently relocated towards the distal cecum. The cecum was ligated at $5 \mathrm{~mm}$ from the distal end and then punctured once with a 21-gauge needle, allowing the exposure of feces (cecal ligation and puncture; CLP). A small droplet of feces was then gently squeezed through both sides of the puncture. The cecum was returned to the peritoneal cavity with careful attention to ensure that feces did not contaminate the margins of the abdominal and skin wound. The muscle and skin incision were closed with 3-0 black silk. This model represents a high-grade sepsis with $100 \%$ mortality in $72 \mathrm{~h}$ (22). Mice received a subcutaneous injection of pre-warmed saline solution $\left(37^{\circ} \mathrm{C} ; 5 \mathrm{ml}\right.$ per $100 \mathrm{~g}$ body weight). Mice receiving sham surgery underwent the same procedure except that the cecum was neither ligated nor punctured (sham group; S). At each experimental time point after performing the procedure, mice were euthanized by cervical dislocation, and brain and skeletal muscle tissue samples were obtained.

Measurement of metabolites. Brain tissues were obtained at $18 \mathrm{~h}$ after the CLP procedure ( $\mathrm{n}=2$ for each group). Approximately $20 \mathrm{mg}$ of frozen brain tissues were immersed into $1,500 \mu \mathrm{l}$ of $50 \%$ acetonitrile/Milli-Q water containing internal standards (H3304-1002; Human Metabolome Technologies, Inc., Tsuruoka, Japan) at $0^{\circ} \mathrm{C}$ in order to inactivate enzymes. The tissue was homogenized three times at $1,500 \mathrm{rpm}$ for $120 \mathrm{~s}$ using a tissue homogenizer (BMS-M10N21; BMS Co., Ltd., Tokyo, Japan) and then the homogenate was centrifuged at $2,300 \mathrm{x} \mathrm{g}$ and $4^{\circ} \mathrm{C}$ for $5 \mathrm{~min}$. Subsequently, $800 \mu \mathrm{l}$ of the upper aqueous layer was centrifugally filtered through a Millipore 5-kDa cut-off filter at 9,100 x g and $4^{\circ} \mathrm{C}$ for $120 \mathrm{~min}$ to remove proteins. The filtrate was centrifugally concentrated and resuspended in $50 \mu \mathrm{l}$ of Milli-Q water for CE-MS analysis. Metabolome measurements were performed by a facility service at Human Metabolome Technology Inc., Tsuruoka, Japan.

Reverse transcription-quantitative polymerase chain reaction $(R T-q P C R)$. Total RNA from the skeletal muscle and brain 


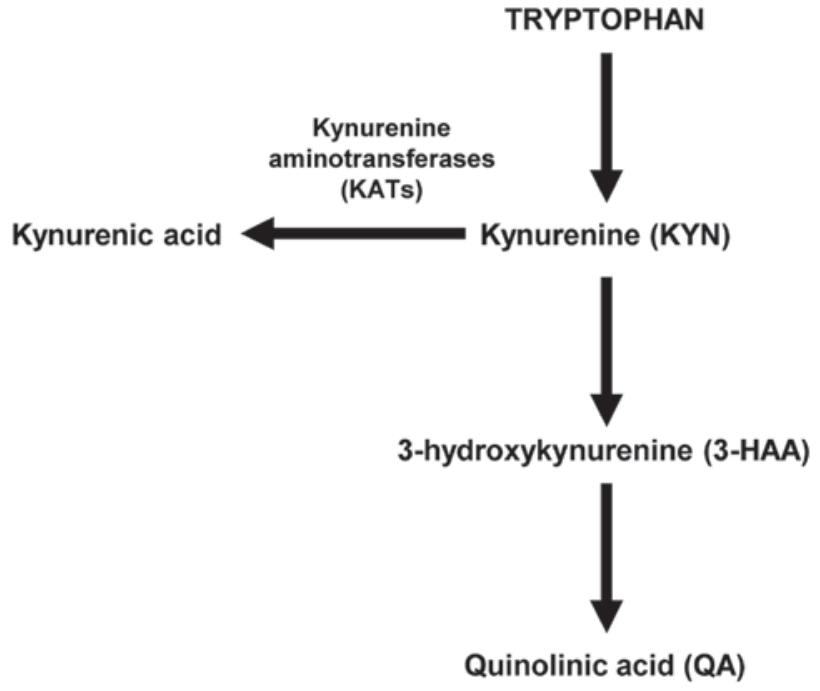

Figure 1. A schematic diagram of the kynurenine pathway.

of the mice was purified by using ISOGEN (Nippon Gene, Tokyo, Japan) according to the manufacturer's instructions and reverse transcribed by using ReverTra Ace with random primers (Toyobo, Osaka, Japan). qPCR was performed by using LightCycler 480 Probes Master (Roche Diagnostics, Mannheim, Germany) and the Step One Plus Detection System (Applied Biosystems, Life Technologies, Tokyo, Japan). The Relative standard curve method (23) was used in this study and expression levels were determined relative to that of 18s rRNA. Primers and probes used in this study are shown in Table I.

Plasmids, siRNA and antibodies. PGV-B2 (PicaGene Basic vector 2; Toyo Ink, Tokyo, Japan) vector and pcDNA3 (Invitrogen; Thermo Fisher Scientific, Inc., Waltham, MA, USA) vector were purchased. pcDNA3 hemagglutinin antigen (HA) was constructed by inserting the HA sequence into pcDNA3 (Invitrogen; Thermo Fisher Scientific, Inc.). The coding sequence of human full-length NRF2 was PCR-amplified (primers, 5'-CAGTGTGCTGGAATTATG ATGGACTTGGAGCTGCC-3' and 5'-GATATCTGCAGA ATTGTTTTTCTTAACATCTGGCTTCTTAC-3') from HeLa cDNA and full-length NRF2 was inserted into the pcDNA3 HA plasmid (Invitrogen; Thermo Fisher Scientific, Inc.) for transient transfection assays. The promoter of the KAT4 gene was PCR-amplified (primers, 5'-AAAGCTAGCAAGCTTCAT ACTGTAAGC-3' and 5'-AAACTCGAGAGAGCCGAGATC TGGGGAAG-3') from the genome of C57BL/6J mice and the fragment $(-2553$ to +30$)$ was subcloned into PGV-B2 (PicaGene Basic vector 2; Toyo Ink). Plasmid sequences were confirmed by sequencing. SYVN1 plasmids and siRNA against Syvn1 were previously described $(9,13)$. The following antibodies were used: Anti-tubulin (Sigma-Aldrich; Merck KGaA, Darmstadt, Germany), and anti-HA (3F10; Roche Diagnostics, Indianapolis, IN, USA), anti-KAT1 and KAT4 (Abcam, Cambridge, UK), anti-KAT2 and KAT3 (Santa Cruz Biotechnology, Inc., Dallas, Texas, USA). The anti-Syvn1 rabbit polyclonal antibody that was used was previously reported (18).

Cell culture and transient transfection. A total of 293 cells and $\mathrm{C} 2 \mathrm{C} 12$ cells were cultured in Dulbecco's modified
Eagle's medium as previously described (9). Transient transfection was performed with Lipofectamine 2000 according to the manufacturer's protocol (Invitrogen; Thermo Fisher Scientific, Inc.). Cells were lysed with cell lysis buffer (Promega Corporation, Madison, WI, USA) $24 \mathrm{~h}$ after transfection, and luciferase activity was measured. To ensure equal amounts of DNA, empty plasmids were added to each transfection.

Luciferase assay. The assay was performed as previously described (24-26). Briefly, 293 cells were transiently transfected with $50 \mathrm{ng}$ KAT4-luc reporter plasmid, $0.1 \mathrm{ng}$ pRL-CMV, and 10, 50, $100 \mathrm{ng}$ pcDNA3 HA-NRF2. For Syvn1 knockdown, 50 ng KAT4-luc reporter plasmid, $0.1 \mathrm{ng}$ pRL-CMV, and 10, $20 \mathrm{nM}$ Syvn1 siRNA were transfected. For SYVN1 overexpression, 50 ng KAT4-luc reporter plasmid, $0.1 \mathrm{ng}$ pRL-CMV, and 10, 50, or $100 \mathrm{ng}$ SYVN1 expression vector were transfected. 293 cells were transiently transfected with $50 \mathrm{ng}$ KAT4-luc reporter plasmid, $0.1 \mathrm{ng}$ pRL-CMV, and 50 ng pcDNA3 HA-NRF2 and/or 25 ng pcDNA3 HA PGC-1 $\beta$. 293 cells were transiently transfected with 50 ng KAT4-luc reporter plasmid, $0.1 \mathrm{ng}$ pRL-CMV, and $50 \mathrm{ng}$ pcDNA3 HA-NRF2 and/or $25 \mathrm{ng}$ pcDNA3 HA PGC-1 $\alpha$. After $24 \mathrm{~h}$, cells were lysed with cell lysis buffer, which was followed by measurement of luciferase activity. Each experiment was performed at least three times.

RNA interference assay. siRNAs for Syvn1 were previously described (9). Transfection with siRNAs $(20 \mu \mathrm{M})$ was performed by using Lipofectamine 2000 (Invitrogen; Thermo Fisher Scientific, Inc.) according to the manufacturer's protocol. Total RNA from $\mathrm{C} 2 \mathrm{C} 12$ cells was purified $3 \mathrm{~d}$ after transfection using ISOGEN (Nippon Gene) according to the manufacturer's instructions, and reverse transcribed using ReverTra Ace with random primers (Toyobo).

Statistical analysis. All data are expressed as the means \pm standard deviation. Differences between two groups were examined by using the Student's t-test and $\mathrm{P}<0.05$ was considered to indicate a statistically significant difference. One-way analysis of variance with Tukey-Kramer post hoc analysis was used to determine correlations in datasets containing multiple groups in luciferase assays. All results were derived from at least three independent experiments.

\section{Results}

Metabolome analysis of Syvn1-deficient septic mice. To investigate the role of Syvn1 in sepsis, we performed the metabolome analysis using brain tissue of WT and heterozygous Syvn1-deficient mice because the homozygous mice die in utero. We first examined the expression of Syvn1 in the brain. Western blotting showed that the expression of Syvn1 was decreased in the heterozygous mice (Fig. 2). As shown in Table II, KYN was not detected in sham mice [WT (sham) and heterozygous Syvn1 mice (sham)] and was increased in CLP-induced septic mice at $18 \mathrm{~h}$ [WT (CLP)]. Interestingly, the quantity of KYN decreased in CLP-induced septic Syvn1-deficient mice [heterozygous Syvn1 mice (CLP)]. This result suggests that decreased expression of Syvn1 might induce KYN metabolism. 
Table I. Primer sequences and length of specific polymerase chain reaction products.

\begin{tabular}{|c|c|c|c|}
\hline Gene & Direction $^{\mathrm{a}}$ & Primer sequence & Probe $^{b}$ \\
\hline \multirow[t]{2}{*}{ Syvnl } & $\mathrm{F}$ & 5'-CTGGGTATCCTGGACTTCCTC-3' & 89 \\
\hline & $\mathrm{R}$ & 5'-AAGCACCATGGTCATCAGAA-3' & \\
\hline \multirow[t]{2}{*}{ KAT1 } & $\mathrm{F}$ & 5'-CAGAGCAGCGCTATTGTTTG-3' & 81 \\
\hline & $\mathrm{R}$ & 5'-GCAGACAGTCTAGGCCAGAAA-3' & \\
\hline \multirow[t]{2}{*}{ KAT3 } & $\mathrm{F}$ & 5'-TTACACGTGTGCGACTCCTT-3' & 27 \\
\hline & $\mathrm{R}$ & 5'-GCTTGATATCGATCCAAAACG-3' & \\
\hline \multirow[t]{2}{*}{ KAT4 } & $\mathrm{F}$ & 5'-ATGGCTGCTGCCTTTCAC-3' & 17 \\
\hline & $\mathrm{R}$ & 5'-GATCTGGAGGTCCCATTTCA-3' & \\
\hline \multirow[t]{2}{*}{$18 s R N A$} & $\mathrm{~F}$ & 5'-GCAATTATTCCCCATGAACG-3' & 48 \\
\hline & $\mathrm{R}$ & 5'-GGGACTTAATCAACGCAAGC-3' & \\
\hline
\end{tabular}

${ }^{\mathrm{a} D i r e c t i o n}$ of primer sequences; ${ }^{b}$ probe number of Universal ProbeLibrary probes (Roche). F, forward; R, reverse; Syvn1, synoviolin; KAT, kynurenine aminotransferase.

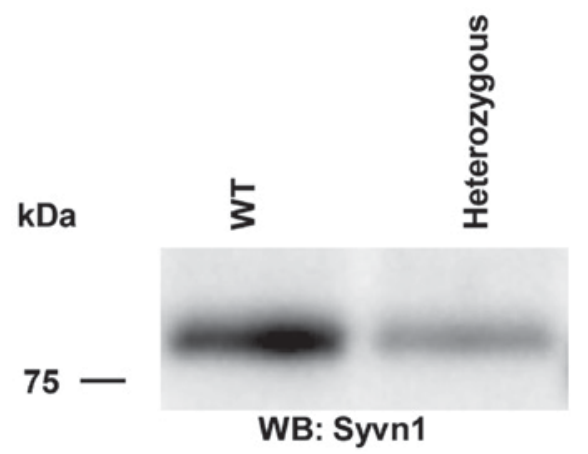

50

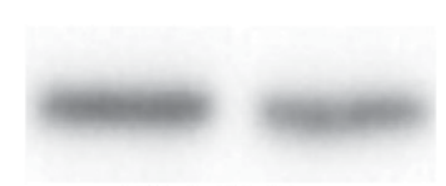

WB: Tubulin

Figure 2. Measurement of kynurenine in Syvn1-deficient mice. Western blot analysis of brain tissue of WT and heterozygous Syvn1-deficient mice using an anti-Syvn1 and anti-tubulin antibodies. Syvn1, synoviolin; WT, wild-type.

Expression of KAT genes in Syvnl-KO mice. The conversion of KYN to KYNA is catalyzed by KATs, which have been detected in the brain and skeletal muscle. Therefore, we examined the expression level of the KATI-4 genes in the brain and the skeletal muscle tissue of tamoxifen (Tam)-inducible Syvn1 knockout (KO) mice (the post-neonatal knockout mice) (9). Real-time PCR assay showed that the expression of Syvnl was very low in the brain tissue of $\mathrm{KO}$ mice and that the expression of KAT3 was significantly increased in KO mice (Fig. 3A). To examine the protein level, we performed western blotting using brain extracts from $\mathrm{KO}$ mice and WT mice. As shown in Fig. 3B, the expression of Syvn1 was very low. However, the expression level of KAT1-4 was similar between WT mice and KO mice. We next investigated the mRNA levels by real-time PCR assay. As shown in Fig. 3C, the expression of Syvnl was very low in the skeletal muscle tissue of KO mice. The expression of KAT4 was significantly increased in $\mathrm{KO}$ mice compared to that in WT mice $(\mathrm{P}<0.05)$, and the expression of
KATI and KAT3 was similar between WT mice and KO mice. $K A T 2$ was not detected in the skeletal muscle tissue. Western blotting using the skeletal muscle extracts revealed that the expression of Syvn1 was very low and that the expression of KAT4 was clearly increased in the skeletal muscle tissue of KO mice (Fig. 3D). These results suggest that KAT4 could be an important gene and Syvn1 might regulate the expression of KAT4.

Syvn1 represses KAT4 expression. To examine the role of Syvn1 in KAT4 expression, we performed luciferase assay using the KAT4 promoter $(-2553 /+30)$ reporter constructs. We used 293 cells, which have high transfection efficiency, because we have used 293 cells as model cells for a long time to analyze transcriptional regulation $(9,25,27)$. As shown in Fig. 4A, treatment with a siRNA against Syvn1 (siSyvn1) induced the luciferase reporter activity, 1.2-fold $(10 \mu \mathrm{M})$ and 1.4-fold $(20 \mu \mathrm{M}$ ), compared to that with control siRNA (siControl). Overexpression of Syvn1 repressed the reporter activity in a dose-dependent manner (Fig. 4B). To further examine whether Syvn1 regulates the KAT4 expression, we performed knock-down assays in $\mathrm{C} 2 \mathrm{C} 12$ cells. $\mathrm{C} 2 \mathrm{C} 12$ cells were treated with control siRNA (siControl) or siRNA for Syvn1 (siSyvn1) for $3 \mathrm{~d}$ and total RNA was purified. Then, we performed RT-PCR and real-time PCR assays to measure the expression of KAT1-4. With siSyvn1 treatment, the expression of Syvn1 decreased to $40 \%$ that of control levels. The expression of KAT4 was significantly increased in cells treated with siSyvn1 compared to that in cells treated with control siRNA (siControl). In addition, the expression of KAT3 and KAT4 was not different between siSyvn1 and siControl conditions (Fig. 4C).

NRF2 and PGC-1 $\beta$ activate the KAT4 promoter. We previously demonstrated that Syvn1 regulates the transcriptional factor, NRF2, and the transcriptional coactivator, PGC-1 $\beta$, which activates NRF2- and PPAR-mediated transcription (12). Therefore, we analyzed the transcriptional factor binding sites of the above factors in the KAT4 promoter $(-2553 /+30)$ using TF BIND (http://tfbind.hgc.jp/). As shown in Fig. 5A, one PPAR responsive element (PRE) and 17 NRF2 binding 
Table II. Metabolome analysis.

\begin{tabular}{|c|c|c|c|c|c|c|c|c|}
\hline \multirow[b]{3}{*}{ Treatment } & \multicolumn{8}{|c|}{ Case } \\
\hline & \multicolumn{2}{|c|}{ WT (sham) } & \multicolumn{2}{|c|}{$\begin{array}{l}\text { Heterozygous } \\
\text { Syvn1 (sham) }\end{array}$} & \multicolumn{2}{|c|}{ WT (CLP) } & \multicolumn{2}{|c|}{$\begin{array}{c}\text { Heterozygous } \\
\text { Syvn1 (CLP) }\end{array}$} \\
\hline & 1 & 2 & 1 & 2 & 1 & 2 & 1 & 2 \\
\hline Kynurenine (nmol/g) & ND & ND & ND & ND & $1.1 \times 10^{-04}$ & $3.1 \times 10^{-04}$ & ND & ND \\
\hline
\end{tabular}

ND, not detected; WT, wild-type; CLP, cecal ligation/perforation.

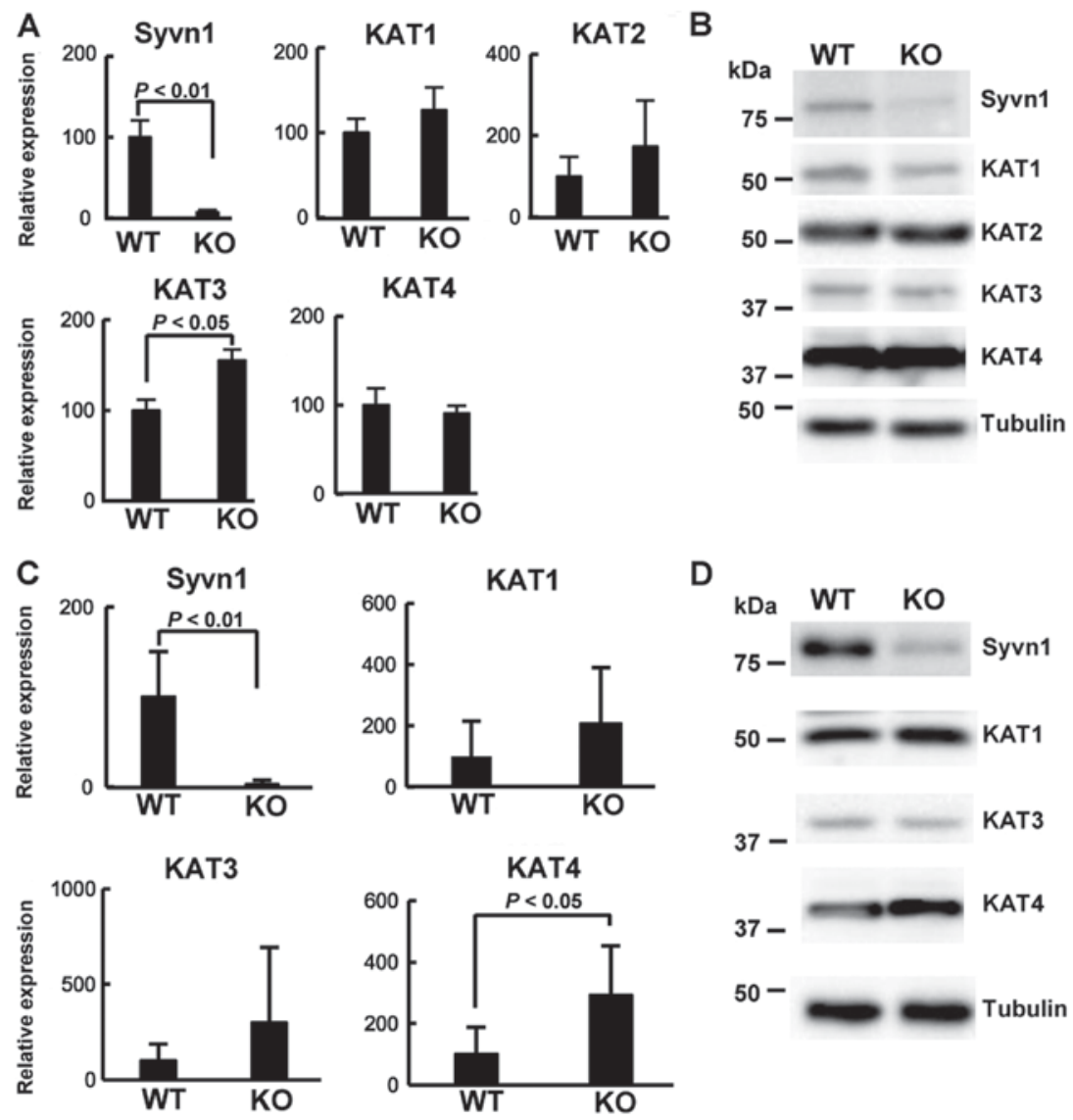

Figure 3. Expression of KAT genes in Syvn1-knockout (KO) mice. mRNA and protein expression of KATs in (A and B) brain and (C and D) skeletal muscle extracts. Total RNA from (A) brain and (C) skeletal muscle was isolated from Syvnl WT (n=8) and Syvn1-KO mice (n=6) and subjected to qPCR. Individual measurements were standardized using 18S RNA, and then the average for Syvnl WT mice was set to 100. Data were analyzed by performing a Student's t-test and expressed as mean \pm SD. The experiment was performed in triplicate. (B) Brain extracts and (D) skeletal muscle extracts were prepared from WT mice and Syvn1-KO mice. Tissue extracts were separated by SDS-PAGE, and processed for western blotting with anti-KAT1, 2, 3, 4, Syvn1 and tubulin antibodies. KAT, kynurenine aminotransferase; Syvn1, synoviolin; WT, wild-type.

sites were detected in the KAT4 promoter. To examine the role of NRF2 and PGC-1 $\beta$, we performed luciferase assays using the KAT4 promoter $(-2553 /+30)$. NRF2 significantly activated the luciferase reporter activity in a dose-dependent manner (Fig. 5B), whereas PPAR- $\alpha$ did not induce the reporter activity (data not shown). As shown in Fig. 5C, NRF2 and PGC-1 $\beta$ synergistically activated the reporter activity. PGC-1 $\alpha$ also induced reporter activity, and NRF2 and PGC- $1 \alpha$ additively activated the reporter activity (Fig. 5D). In addition, NRF2 rescued the repressive effect induced by overexpression of Syvn1 in a dose-dependent manner (Fig. 5E). These results suggest that the NRF2-PGC-1 $\beta$ pathway induces the expression of KAT4.

\section{Discussion}

In the present study, we investigated the role of Syvn1 in a mouse model of sepsis. We showed that the level of KYN was elevated in the brain tissue of septic WT mice. However, KYN was not detected in septic Syvn1-deficient mice. In addition, expression of KAT4, which encodes one of the enzymes that converts KYN into KYNA, was elevated in the skeletal muscle 

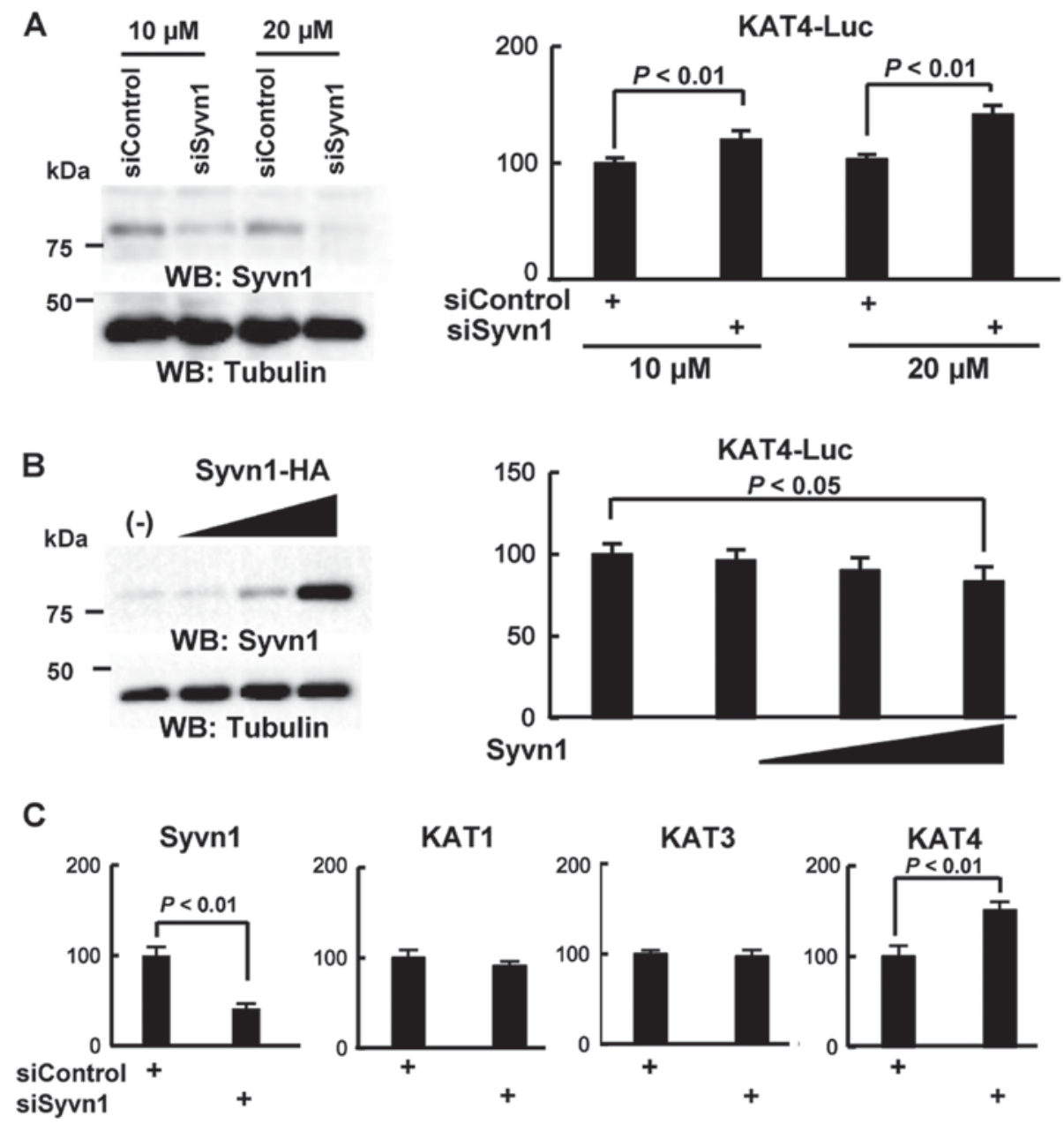

Figure 4. Syvn1 represses KAT4 expression based on KAT4 promoter-driven luciferase reporter assays. (A) Effect of Syvn1 knockdown by siRNA. 293 cells were transiently transfected with a reporter plasmid containing the KAT4 promoter, pRL-CMV, control siRNA (siControl) or siRNA for Syvn1 (siSyvn1). Data were analyzed by a Student's t-test and expressed as mean \pm SD. Western blotting was performed with anti-Syvn1 and anti-tubulin antibodies. (B) Effect of Syvn1 overexpression. 293 cells were transiently transfected with a reporter plasmid containing the KAT4 promoter, pRL-CMV, and the HA-tagged Syvn1 (Syvn1-HA)-expression vector (10, 50, and $100 \mathrm{ng}$ ). Analysis of variance with Tukey-Kramer post hoc analysis and expressed as mean \pm SD. Western blotting was performed with anti-Syvn1 and anti-tubulin antibodies. (C) Effect of Syvn1 on KAT4 expression. C2C12 cells were transiently transfected with siControl or siSyvn1. After 3 days, total RNA were purified and qPCR was performed. Individual measurements were standardized using 18S RNA, and the average for siControl was set to 100. Data were analyzed by performing a Student's t-test and expressed as mean \pm SD. The experiment was performed three times. KAT, kynurenine aminotransferase; Syvn1, synoviolin; HA, hemagglutinin antigen.

tissue of Syvn1-KO mice. Moreover, Syvn1 knockdown induced KAT4 promoter-driven reporter activity, whereas overexpression of Syvn1 repressed this effect. The KAT4 promoter was also activated by the NRF2-PGC-1 $\beta$ pathway. NRF2 and PGC-1 $\beta$ are target proteins of Syvn1-induced degradation $(9,17)$. Taken together, these results suggest that Syvn1 deficiency might induce KYN metabolism via the NRF2-PGC-1 $\beta$-KAT4 pathway.

KATs are critical enzymes that catalyze the conversion of KYN to KYNA. Recent studies indicates that exercise induces the expression of KATs expression in the skeletal muscle $(7,8,28)$. Analysis of skeletal muscle-specific PGC-1 $\alpha$-transgenic mice revealed that an increased expression of KATs in skeletal muscle shifts the KYN metabolism towards enhanced synthesis of KYNA, resulting in a decrease in KYN levels, and thereby protecting the tissue from stress-induced damage. This novel function of PGC-1 $\alpha$ in the regulation of the KYN metabolism suggests communication between the skeletal muscle and brain. In the present study, we showed that KAT4 expression was significantly increased in the skeletal muscle tissue of Syvn1-deficient mice and that the NRF2-PGC-1 $\beta$ pathway activates the KAT4 promoter. We previously demonstrated that Syvn1 interacts with NRF2 and PGC-1 $\beta$, and induces the degradation of these factors through ubiquitination. Therefore, in Syvn1-deficient mice, accumulated NRF2 and PGC-1 $\beta$ could activate KAT4 expression in the skeletal muscle to enhance the synthesis of KYNA, resulting in decreased KYN in the brain tissue.

Syvn1 is involved in both acute and chronic inflammation. We previously demonstrated that Syvn1 overexpression in transgenic mice led to advanced arthropathy through the suppression of apoptosis in synoviocytes and that heterozygous Syvn1-mice were resistant to arthritis and fibrosis $(13,15)$. In addition, we recently showed that Syvn1 is involved in the development of obesity, limb girdle muscular dystrophy, and liver cirrhosis. These studies indicate that Syvn1 is a key factor for diseases associated with chronic inflammation. In the present study, we examined the role of Syvn1 in a CLP-induced 
A

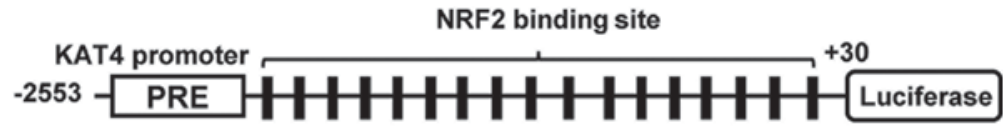

B

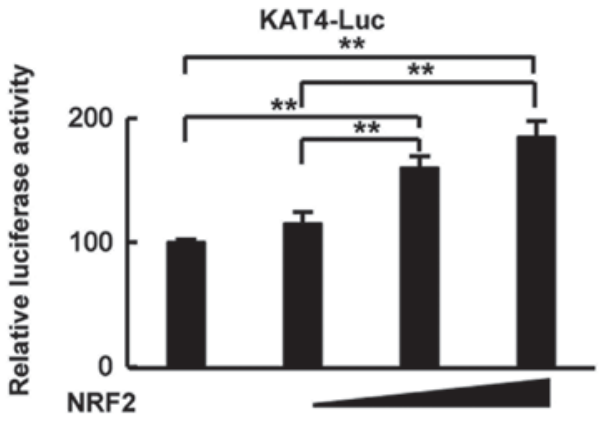

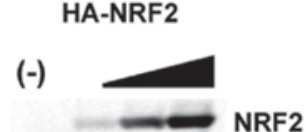

WB: anti-HA

C
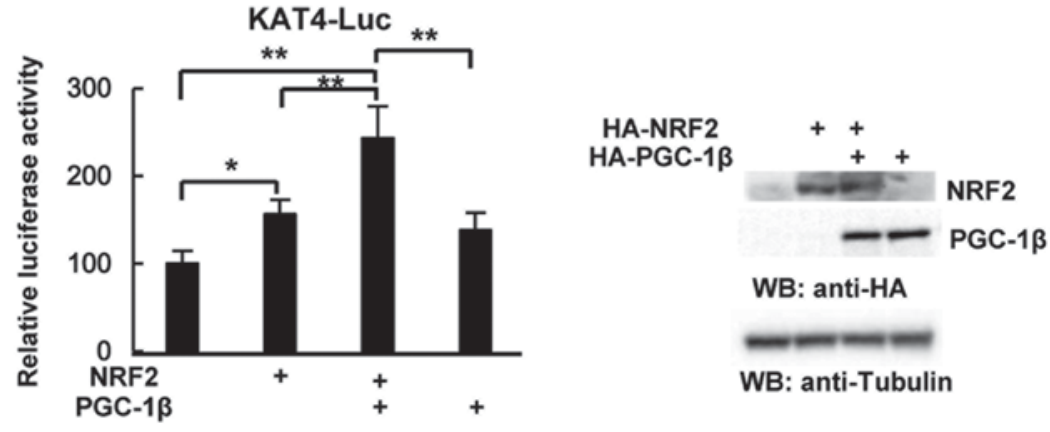

WB: anti-HA

WB: anti-Tubulin

D

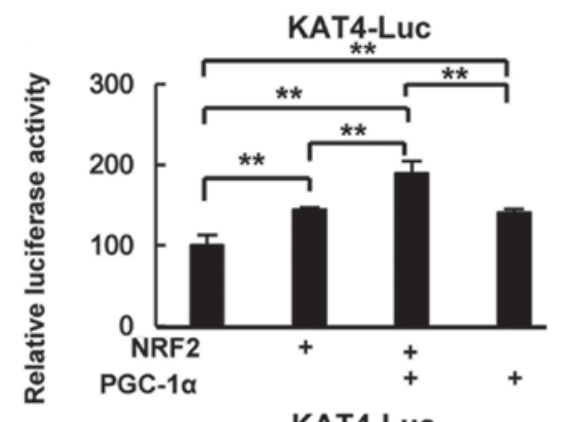

$\mathbf{E}$
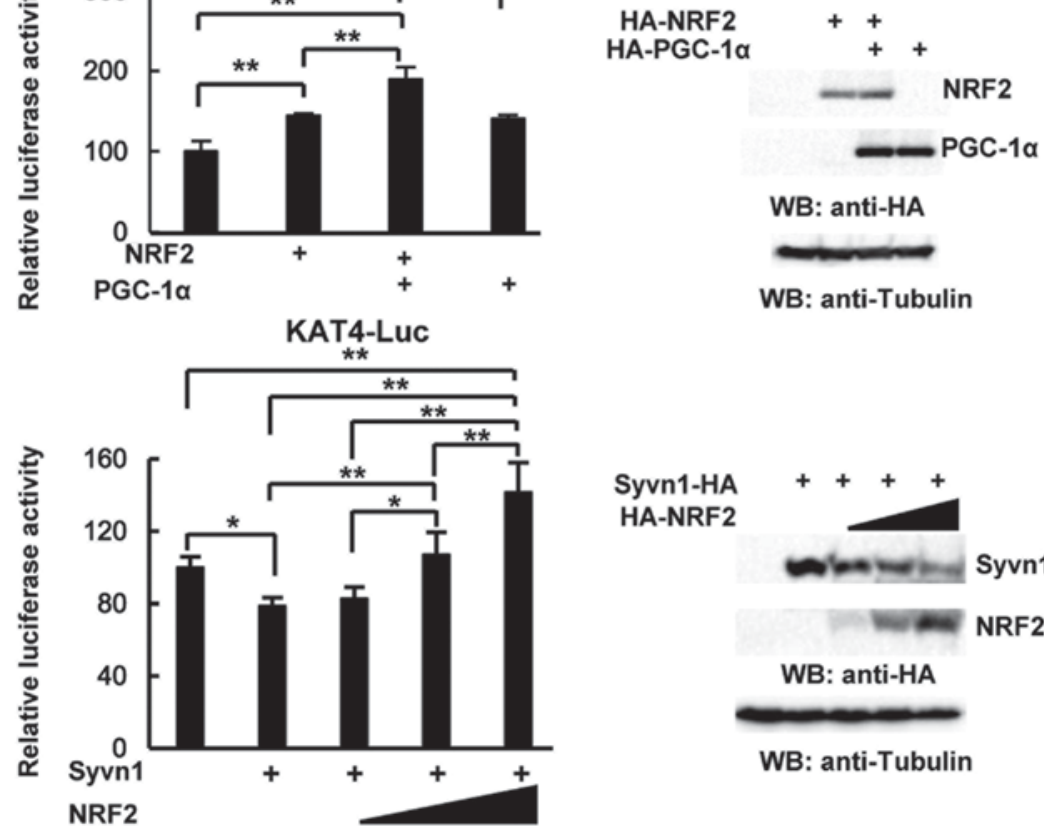

WB: anti-HA

WB: anti-Tubulin

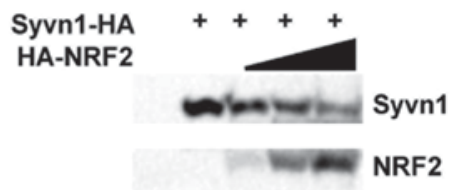

WB: anti-HA

WB: anti-Tubulin

Figure 5. The NRF2/PGC-1 $\beta$ pathway activates the KAT4 promoter. (A) Schematic representation of the KAT4 promoter (-2553/+30). PRE: PPAR responsive element, black box: NRF2 binding site $(-2123 /-2114,-2029 /-2020,-1981 /-1972,-1957 /-1948,-1742 /-1733,-1732 /-1723,-1459 /-1450,-1386 /-1377,-1378 /-1369$, $-1308 /-1299,-1106 /-1097,-759 /-750,-359 /-350,-250 /-241,-105 /-96,-46 /-37$, and +2/+11). (B) Effect of NRF2 overexpression on KAT4 promoter-driven luciferase reporter activity. 293 cells were transiently transfected with a reporter plasmid containing the KAT4 promoter, pRL-CMV, and the HA-NRF2-expression vector $(10,50$, and $100 \mathrm{ng})$. Western blotting was performed with an anti-HA and anti-tubulin antibodies. (C) Effect of NRF2 and PGC-1 $\beta$ overexpression on the KAT4 promoter-driven luciferase reporter activity. 293 cells were transiently transfected with a reporter plasmid containing the KAT4 promoter, pRL-CMV, $50 \mathrm{ng}$ of the HA-NRF2-expression vector, and $25 \mathrm{ng}$ of the HA-PGC-1 $\beta$-expression vector. Western blotting was performed with an anti-HA and anti-tubulin antibodies. (D) Effect of NRF2 and PGC-1 $\alpha$ overexpression on KAT4 promoter-driven luciferase reporter activity. A total of 293 cells were transiently transfected with a reporter plasmid containing the KAT4 promoter, pRL-CMV, $50 \mathrm{ng}$ of the HA-NRF2-expression vector, and $25 \mathrm{ng}$ of the HA-PGC-1 $\alpha$-expression vector. Western blotting was performed with an anti-HA and anti-tubulin antibodies. (E) NRF2 overexpression abrogates the effect of Syvn1 overexpression on KAT4 promoter-driven luciferase reporter activity. 293 cells were transiently transfected with a reporter plasmid containing the KAT4 promoter, pRL-CMV, $100 \mathrm{ng}$ of the Syvn1-HA-expression vector, and the HA-NRF2-expression vector (10, 50, and $100 \mathrm{ng})$. Western blotting was performed with an anti-HA and anti-tubulin antibodies. (B-D) Data were analyzed by performing a Tukey-Kramer post hoc analysis and expressed as mean \pm SD $\left({ }^{*} \mathrm{P}<0.05,{ }^{* *} \mathrm{P}<0.01\right)$. The experiment was performed three times. KAT, kynurenine aminotransferase; Syvn1, synoviolin; HA, hemagglutinin antigen; PGC, proliferator-activated receptor (PPAR)-PPAR coactivator. 
septic mouse model, which is an example of acute inflammation. Proinflammatory cytokines such as tumor necrosis factor (TNF) $-\alpha$ and interleukin (IL) $-1 \beta$ are induced in the mouse sepsis model and septic patients $(5,29,30)$. Previous studies demonstrated that Syvn1 is a key target for inflammatory cytokines such as TNF- $\alpha$, IL-1, and IL-17 $(5,31,32)$, and that the expression of Syvn1 is transcriptionally regulated by Ets transcription factors, GABP $\alpha$, GABP $\beta$, and ILF-3 $(33,34)$, which are the downstream of inflammatory cytokines signaling. These results prompted us to speculate that Syvn1 expression might be induced by inflammatory cytokines during sepsis and induce the degradation of NRF2 and PGC-1 $\beta$ in the skeletal muscle tissue. Further studies are warranted to unveil the detailed role of Syvn1 in sepsis.

KYN is a key immune mediator. During inflammation, increases in cytokines such as interferon (IFN)- $\gamma, \mathrm{TNF}-\alpha$, and IL-1 $\beta$ activate indoleamine 2,3-dioxygenase (IDO), and tryptophan is metabolized into the toxic metabolite $\mathrm{KYN}$ via IDO. Several studies indicate that the development of mood symptoms with inflammation is associated with reduced circulating tryptophan levels and concomitant increases in serum levels of KYN $(35,36)$. In addition, recent studies show that KYN and proinflammatory cytokines such as TNF- $\alpha$ and IL-1 $\beta$ are induced in mouse sepsis models and human septic patients $(5,29,30)$. Therefore, the KYN pathway is one of targets for the treatment of SAE. The IDO inhibitor, 1-methyl-D, was shown to attenuate neuroinflammation through the repression of proinflammatory cytokines and $\mathrm{KYN}$, and protect against sepsis-induced cognitive impairment in a mouse model (5). In the present study, we showed that the induction of KYN by CLP is not observed in Syvn1-deficient mice. We previously developed a Syvn1 inhibitor, LS-102, and demonstrated that the Syvn1 inhibitor attenuated arthritis, fibrosis, obesity, limb girdle muscular dystrophy, and liver cirrhosis (13-17). Although further studies are needed to investigate the effect of this Syvn1 inhibitor on SAE, Syvn1 might represent a novel target for the treatment of SAE.

Taken together, in the present study, we provide evidence that Syvn1 regulates the NRF2-PGC-1 $\beta$-KAT4 pathway in a mouse model of sepsis. Further analysis of Syvn1 will be helpful to understand its physiological and clinical significance.

\section{Acknowledgements}

The authors thank Mr. Shyouichiro Shibata (Institute of Medical Science, Tokyo Medical University) for technical assistance. We also thank all members of Professor Toshihiro Nakajima's laboratory (Institute of Medical Science, Tokyo Medical University).

\section{Funding}

This study was funded in part by grants from the Naito Foundation, Natural Science Scholarship Daiichi-Sankyo Foundation of Life Science, Mitsubishi, Tanabe Pharma Corporation, Bureau of Social Welfare and Public Health, Academic contribution of Pfizer, Eisai, Santen Pharmaceutical, Abbvie, Astellas, Takeda Science Foundation, AstraZeneca (R\&D Grant 2013), and ONO Medical Research Foundation. This study was supported in part by funds provided through a MEXT-Supported program of the Strategic Research Foundation at Private Universities (grant no. S1411011, 2014-2018) from the Ministry of Education, Culture, Sports, Science and Technology of Japan. This study was also supported in part by the Japan Society for the Promotion of Science KAKENHI (grant nos. 23659176, 26670479, 26461478 and 16H05157) and Industry-university cooperation (BioMimetics Sympathies Inc.).

\section{Availability of data and materials}

All data generated or analyzed during this study are included in this published article.

\section{Authors' contributions}

YI, HF, HU and TN conceived the project and designed the experiments. YI, MC, NT, YO, NU, FN and HU performed the sepsis model and analyzed the metabolome data. YI, HF, SA, MY and TN performed experiments and analyzed data. YI, HF and TN wrote the manuscript. YI, HF, SA, MC, NT, MY, YO, NU, FN, HU and TN discussed the results and commented on the manuscript.

\section{Ethics approval and consent to participate}

All procedures involving animals were performed in accordance with institutional and national guidelines for animal experimentation, and were approved by the Institutional Animal Care and Use Committee of Tokyo Medical University (approval no. S-28044).

\section{Consent for publication}

Not applicable.

\section{Competing interests}

The authors declare that they have no competing interests.

\section{References}

1. Sprung CL, Peduzzi PN, Shatney CH, Schein RM, Wilson MF, Sheagren JN and Hinshaw LB: Impact of encephalopathy on mortality in the sepsis syndrome. The Veterans Administration Systemic Sepsis Cooperative Study Group. Crit Care Med 18: 801-806, 1990.

2. Eidelman LA, Putterman D, Putterman C and Sprung CL: The spectrum of septic encephalopathy. Definitions, etiologies, and mortalities. JAMA 275: 470-473, 1996.

3. Zwilling D, Huang SY, Sathyasaikumar KV, Notarangelo FM, Guidetti P, Wu HQ, Lee J, Truong J, Andrews-Zwilling Y, Hsieh EW, et al: Kynurenine 3-monooxygenase inhibition in blood ameliorates neurodegeneration. Cell 145: 863-874, 2011.

4. Stone TW and Darlington LG: The kynurenine pathway as a therapeutic target in cognitive and neurodegenerative disorders. Br J Pharmacol 169: 1211-1227, 2013.

5. Gao R, Kan MQ, Wang SG, Yang RH and Zhang SG: Disrupted tryptophan metabolism induced cognitive impairment in a mouse model of sepsis-associated encephalopathy. Inflammation 39: 550-560, 2016.

6. Moroni F, Carpenedo R, Cozzi A, Meli E, Chiarugi A and Pellegrini-Giampietro DE: Studies on the neuroprotective action of kynurenine mono-oxygenase inhibitors in post-ischemic brain damage. Adv ExpMed Biol527: 127-136,2003. 
7. Agudelo LZ, Femenia T, Orhan F, Porsmyr-Palmertz M, Goiny M, Martinez-Redondo V, Correia JC, Izadi M, Bhat M, Schuppe-Koistinen I, et al: Skeletal muscle PGC-1 $\alpha 1$ modulates kynurenine metabolism and mediates resilience to stress-induced depression. Cell 159: 33-45, 2014.

8. Ruas JL, White JP, Rao RR, Kleiner S, Brannan KT, Harrison BC, Greene NP, Wu J, Estall JL, Irving BA, et al: A PGC-1 $\alpha$ isoform induced by resistance training regulates skeletal muscle hypertrophy. Cell 151: 1319-1331, 2012.

9. Fujita H, Yagishita N, Aratani S, Saito-Fujita T, Morota S, Yamano Y, Hansson MJ, Inazu M, Kokuba H, Sudo K, et al: The E3 ligase synoviolin controls body weight and mitochondrial biogenesis through negative regulation of PGC-1 $\beta$. EMBO J 34 1042-1055, 2015

10. Kressler D, Schreiber SN, Knutti D and Kralli A: The PGC-1-related protein PERC is a selective coactivator of estrogen receptor alpha. J Biol Chem 277: 13918-13925, 2002.

11. Lin J, Puigserver P, Donovan J, Tarr P and Spiegelman BM: Peroxisome proliferator-activated receptor gamma coactivator 1beta (PGC-1beta), A novel PGC-1-related transcription coactivator associated with host cell factor. J Biol Chem 277 1645-1648, 2002.

12. Scarpulla RC: Nuclear control of respiratory gene expression in mammalian cells. J Cell Biochem 97: 673-683, 2006.

13. Amano T, Yamasaki S, Yagishita N, Tsuchimochi K, Shin H, Kawahara K, Aratani S, Fujita H, Zhang L, Ikeda R, et al: Synoviolin/Hrd1, an E3 ubiquitin ligase, as a novel pathogenic factor for arthropathy. Genes Dev 17: 2436-2449, 2003.

14. Bianchini E, Fanin M, Mamchaoui K, Betto R and Sandonà D: Unveiling the degradative route of the V247M $\alpha$-sarcoglycan mutant responsible for LGMD-2D. Hum Mol Genet 23: 3746-3758, 2014

15. Hasegawa D, Fujii R, Yagishita N, Matsumoto N, Aratani S, Izumi T, Azakami K, Nakazawa M, Fujita H, Sato T, et al: E3 ubiquitin ligase synoviolin is involved in liver fibrogenesis. PLoS One 5: e13590, 2010.

16. Li L, Shen Y, Ding Y, Liu Y, Su D and Liang X: Hrd1 participates in the regulation of collagen I synthesis in renal fibrosis. Mol Cell Biochem 386: 35-44, 2014.

17. Wu T, Zhao F, Gao B, Tan C, Yagishita N, Nakajima T, Wong PK, Chapman E, Fang D and Zhang DD: Hrd1 suppresses Nrf2-mediated cellular protection during liver cirrhosis. Genes Dev 28: 708-722, 2014.

18. Yamasaki S, Yagishita N, Sasaki T, Nakazawa M, Kato Y, Yamadera T, Bae E, Toriyama S, Ikeda R, Zhang L, et al: Cytoplasmic destruction of p53 by the endoplasmic reticulum-resident ubiquitin ligase 'Synoviolin'. EMBO J 26: 113-122, 2007.

19. Buendia I, Michalska P, Navarro E, Gameiro I, Egea J and León R: Nrf2-ARE pathway: An emerging target against oxidative stress and neuroinflammation in neurodegenerative diseases. Pharmacol Ther 157: 84-104, 2016.

20. Chen PC, Vargas MR, Pani AK, Smeyne RJ, Johnson DA, Kan YW and Johnson JA: Nrf2-mediated neuroprotection in the MPTP mouse model of Parkinson's disease: Critical role for the astrocyte. Proc Natl Acad Sci USA 106: 2933-2938, 2009.

21. Innamorato NG, Rojo AI, García-Yagüe AJ, Yamamoto M, de Ceballos ML and Cuadrado A: The transcription factor Nrf2 is a therapeutic target against brain inflammation. J Immunol 181: 680-689, 2008

22. Rittirsch D, Huber-Lang MS, Flierl MA and Ward PA: Immunodesign of experimental sepsis by cecal ligation and puncture. Nat Protoc 4: 31-36, 2009.
23. Livak K: ABI Prism 7700 Sequence Detection System, User Bulletin 2. PE Applied Biosystems, Foster City, CA. 1997.

24. Chakravarti D, LaMorte VJ, Nelson MC, Nakajima T, Schulman IG, Juguilon H, Montminy $M$ and Evans RM: Role of CBP/P300 in nuclear receptor signalling. Nature 383: 99-103, 1996.

25. Fujita H, Fujii R, Aratani S, Amano T, Fukamizu A and Nakajima T: Antithetic effects of MBD2a on gene regulation. Mol Cell Biol 23: 2645-2657, 2003.

26. Nakajima T, Fukamizu A, Takahashi J, Gage FH, Fisher T, Blenis J and Montminy MR: The signal-dependent coactivator CBP is a nuclear target for pp90RSK. Cell 86: 465-474, 1996

27. Fujita H, Ohshima T, Oishi T, Aratani S, Fujii R, Fukamizu A and Nakajima T: Relevance of nuclear localization and functions of RNA helicase A. Int J Mol Med 15: 555-560, 2005.

28. Schlittler M, Goiny M, Agudelo LZ, Venckunas T, Brazaitis M, Skurvydas A, Kamandulis S, Ruas JL, Erhardt S, Westerblad H and Andersson DC: Endurance exercise increases skeletal muscle kynurenine aminotransferases and plasma kynurenic acid in humans. Am J Physiol Cell Physiol 310: C836-C840, 2016.

29. Schäfer ST, Franken L, Adamzik M, Schumak B, Scherag A, Engler A, Schönborn N, Walden J, Koch S, Baba HA, et al: Mitochondrial DNA: An endogenous trigger for immune paralysis. Anesthesiology 124: 923-933, 2016

30. Darcy CJ, Davis JS, Woodberry T, McNeil YR, Stephens DP, Yeo TW and Anstey NM: An observational cohort study of the kynurenine to tryptophan ratio in sepsis: Association with impaired immune and microvascular function. PLoS One 6: e21185, 2011.

31. Toh ML, Gonzales G, Koenders MI, Tournadre A, Boyle D, Lubberts E, Zhou Y, Firestein GS, van den Berg WB and Miossec P: Role of interleukin 17 in arthritis chronicity through survival of synoviocytes via regulation of synoviolin expression. PLoS One 5: e13416, 2010.

32. Toh ML, Marotte H, Blond JL, Jhumka U, Eljaafari A, Mougin B and Miossec P: Overexpression of synoviolin in peripheral blood and synoviocytes from rheumatoid arthritis patients and continued elevation in nonresponders to infliximab treatment. Arthritis Rheum 54: 2109-2118, 2006.

33. Izumi $\mathrm{T}$, Fujii R, Izumi $\mathrm{T}$, Nakazawa $\mathrm{M}$, Yagishita $\mathrm{N}$ Tsuchimochi K, Yamano Y, Sato T, Fujita H, Aratani S, et al: Activation of synoviolin promoter in rheumatoid synovial cells by a novel transcription complex of interleukin enhancer binding factor 3 and GA binding protein alpha. Arthritis Rheum 60: 63-72, 2009.

34. Tsuchimochi K, Yagishita N, Yamasaki S, Amano T, Kato Y, Kawahara K, Aratani S, Fujita H, Ji F, Sugiura A, et al: Identification of a crucial site for synoviolin expression. Mol Cell Biol 25: 7344-7356, 2005.

35. Heisler JM and O'Connor JC: Indoleamine 2,3-dioxygenase-dependent neurotoxic kynurenine metabolism mediates inflammation-induced deficit in recognition memory. Brain Behav Immun 50: 115-124, 2015.

36. Maes M, Leonard BE, Myint AM, Kubera M and Verkerk R: The new '5-HT' hypothesis of depression: Cell-mediated immune activation induces indoleamine 2,3-dioxygenase, which leads to lower plasma tryptophan and an increased synthesis of detrimental tryptophan catabolites (TRYCATs), both of which contribute to the onset of depression. Prog Neuropsychopharmacol Biol Psychiatry 35: 702-721, 2011. 